rung an die Dracaenoideae, wohin bisher nur Sansevieria gestellt worden war, Ophiopogon und einige weitere Gattungen jedoch nicht, befürwortet. Es mag vielleicht kein Zufall sein, daß die Dracaenoideae im System den Asparagoideae vorangehen. Wir haben heute gewiß keinen morphologischen Grund, an der Blattnatur der Assimilationsorgane von Dracaena, Ophiopogon und Sansevieria zu zweifeln; wohl aber löst der Vergleich mit Myrsiphyllum weitere Erwägungen aus: wir müssen zugeben, daß wir hier auf einem Grenzgebiete stehen, auf dem die Entscheidung über die morphologische Bedeutung der Assimilationsorgane äußerst schwer geworden ist. Bei den Phyllokladien der Asparageen haben wir noch die Kaulomnatur nachweisen können, bei Dracaena, Ophiopogon, Sansevieria sind jedoch diese Bildungen schon morphologisch so sehr verwischt, daß wir keine Anhaltspunkte mehr finden, die Assimilationsorgane anders als für Blätter zu erklären, wenngleich die vergleichende Anatomie auf eine andere Auffassung hinzuweisen scheint. Auf weitere Erörterungen wollen wir vorläufig verzichten. Jedenfalls ist der Bau der Assimilationsorgane von Myrsiphyllum ein wertvoller Anhaltspunkt für die Beziehungen zur vorhergehenden Unterfamilie.

Folgendes Schema soll die phylogenetischen Beziehungen zwischen den Gattungen der Asparageen veranschaulichen, soweit sich der Nervenbau hatte verwenden lassen.

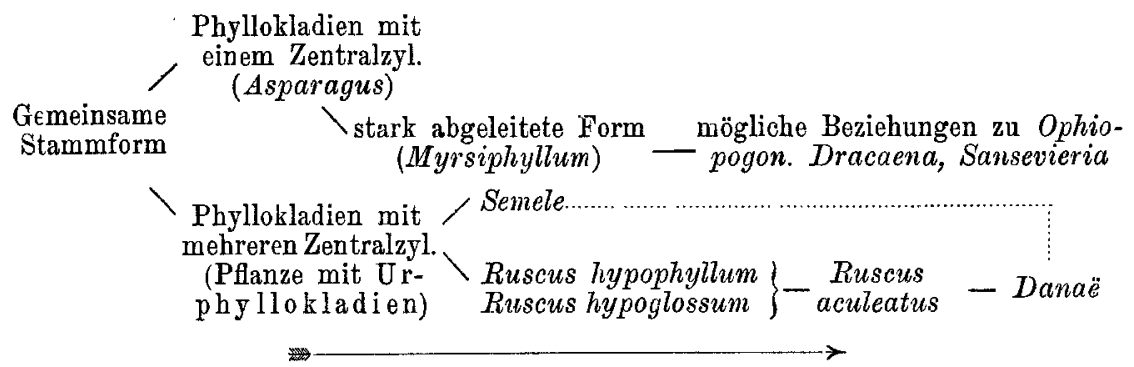

\title{
Verzeichnis der von mir gemachten Angaben zur Systematik und Synonymio der Pilze.
}

Von Prof. Dr. Franz von Höhnel (Wien).

(Fortsetzung. ${ }^{1}$ )

790. Ophiodothella tarda (Harkn.) v. H. (XLIV, 941) = Ophiodothis tarda Harkn.

791. Ophiodothella Ulei (Rehm) v. H. (XLIV, 940) = Ophiodothis Ulei Rehm.

792. Ophiodictyon Sacc. et Syd. (XLVIII, 418) ist eine HypocreaceenGattung.

S. $374-389$. 
793. Ophionectria ? Puiggari Speg. 1889 (LII, 349, 35̃1) = Nectria abnormis P. Henn. $1897=$ Nectria (Dialonectria) Leguminum Rehm 1900.

794. Ophiosphaeria Kirchstein $(\mathrm{XXV}, 642)=$ Ophiochaeta Berl.

795. Ophiosphaeria tenella Kirchst. $(\mathrm{XXV}, 642)=$ Opiochaeta chaetophora (Cr.) Sace.

796. Orbilia flavide-roseola Rehm (XIX, 1276) = Orbilia flavida Feltg.

797. Otthia aceris Wint. (XIX, 1206) $=$ Massariella acerina Sace. et Syd.

798. Otthia bertioides (Sace. et Berl.) v. H. (XLVIII, 426) = Lizonia bertioides Sacc. et Berl.

799. Otthia ? connata (Berk: et Curt.) v. H. vel n. G. (XLI, 415) = Asterina connata $\mathrm{B}$. et $\mathrm{C}$.

800. Otthia Selaginellae (Rac.) v. H. (XLVIII, 426) = Lizonia Selaginellae Raciborski.

801. Otthia Smilacis (Rac.) v. H. (XLVIII, 426) = Lizonia Smilacis Raciborski.

802. Otthia (Otthiella ?) Syzygii (Rac.) v. H. (XLVIII, 428) = Lizonia Syzygii Racib.

803. Otthia Uleana (Sacc. et Syd.) จ. H. (XLVIII, 427) = Lizonia Uleana Sace. et Sydow.

804. Otthiella? conglobata (B. et C.) v. H. vel n. G. $(X L I, 416)=$ Asterina conglobata B. et Curt.

805. Otthiella Leguminis (Rehm) (XLVIII, 430) = Lizonia (Lizoniella) Leguminis Rehm.

806. Otthiella paraguayensis (Speg.) จ H. (XLVIII, 429) = Lizonia? paraguayensis Speg. = Nectria lizonoides v. $\mathrm{H} .=$ Lizonia (Lizoniella) Perkinsiae P. Henn.

807. Otthiella Tournefortiae (Rehm) v. H. (XLVIII, 427) = Lizonia (Lizoniella) Vleana Sace. et Syd. forma Tournefortiae Rehm.

808. Oudemansiella Speg. (XLIV, 885) $=$ Phaeolimasium P. Henn. = Mucidula Pat.

809. Oudemansiella Canarii (Jungh.) v. H. (XXXIII, 1003; XXXV, $275 ; \mathrm{XXXXIV}, 885)=$ Agaricus Canarii Jungh. $=$ Agaricus (Collybia) apalosarcus B. et Br. = Agaricus (Collybia) Magisterium B. et Br. = Agaricus (Collybia) euphylla B. et Br. = Amanitopsis Canarii (Jungh.) Sace. = Phaeolimacium bulbosum P. Henn. $=$ Pluteus macrosporus P. Henn. = Oudemansiella apalosarca (B. et Br.) v. H.

810. Oudemansiella cheimonophylla (Berk. et Curt.) จ. H. (XLIV, 885) $=$ Agaricus (Armillaria) cheimonophyllus B. et C. = Oudemansiella platensis Speg.

811. Oudemansiella mucida (Schrad.) จ. H. (XLIV, 885) $=$ Agaricus mucidus Schrad. = Armillaria mucida (Schr.) Fr.

812. Parenglerula Mac-Owaniana (Thüm.) ซ. H. (XLI, 465) $=$ Meliola Mac-Owaniana Thüm. = Asterina Mac-Owaniana $\mathrm{K}$. et $\mathrm{C}$.

813. Patellea sanguinea (F.) $(\mathrm{XXXV}, 381)=$ Tapesia cruenta P. Henn. et Ploettn. 
814. Patellaria proxima B. et $\mathrm{Br}$. (XIX, 1260, 1266) $=$ Pseudographis hysterioides Feltg. $=$ Pseudographis Mahoniae Feltg. $=$ Leciographa patellarioides Feltg.

815. Patinella punctiformis Rehm forma lignicola (XIX, 1266) $=$ Patinella punctiformis Rehm f. quercina Feltg.

816. Patoullardiella quercicola (P. H.) v. H. (XLVIII, 405) $=$ Auerswaldiopsis quercicola P. Henn.

817. Paxillus (Tapinia) lamellirugis D. C. var. ionipus Oud. (LII, $346)=$ Panus chochlearis Oudem.

818. Peckiella boleticola (Schw.) v. Höhnel (LII, 356) $=$ Sphaeria (Byssisedae) boleticola Schw.

819. Peltistroma P. Henn. (XLIV, 946) von Phragmopeltis P. Henn. ch. em. v. Höhn. kaum verschieden.

820. Peltosphaeria Cerei (P. H.) จ. Н. (XXXVI, 872) = Diplotheca Cerei P. Henn.

821. Pemphidium Montagne (X.LVIII, 435) ist eine Sphaeriaceen-Gattung mit Physalospora verwandt.

822. Peniophora Aegerita (Hoffm.) v. H. et L. (XXVIII, 814) = Corticium lacteum Fuckel.

823. Peniophora byssoidea (P.) v. H. et L. (XXI, 1573; XXXI, 9; XXXII, 1084) $=$ Corticium lacunosum B. et Br. = Tomentella obducens Karst.

824. Peniophora caesia (Bres.) v. H. et L. (XXI, 1593) $=$ Thelephora Lycii P. var. a. lilacea Desm. Pl. Crypt., $119=$ Thelephora cinerea Fries v. Tiliae Desm. Pl. Crypt., 666 .

825. Peniophora cinerea (Fr.) Cooke (XXVIII, 762, XXI, 1579) = Corticium quercinum Fries var. tiliaceum Thüm. $=X y$ lobolus tumulosus Karst.

826. Peniophora citrina P. Henn. (XXVIII, 746) ist zu streichen.

827. Peniophora convolvens (K.) v. H. et L. $(\mathrm{XXI}, 1551)=$ Corticium convolvens Karst.

828. Peniophora corticalis (Bull.) Bres. var. Komabensis (P. H.) v. H. et L. (XXVIII, 744) = Corticiun Komabense P. Henu.

829. Peniophora crocea (Karst.) v. H. et L. (XXI, 1574) = Coniophora crocea Karst. $=$ Xerocarpus laeticolor Karst.

830. Peniophora Dussi Pat. (XXVIII, 749) $=$ Hypochnus Dussi Pat.

831. Peniophora fumigata (Thüm.) v. H. et L. (XXVIII, 782) $=$ Corticium fumigatum Thüm.

832. Peniophora glebulosa (Fr.) Sace. et Syd. (XXVIII, 743) = Peniophora gracillima $\mathrm{E}$. et Ev.

833. Peniophora Kalchbrenneri (Massee) v. H. et L. (XXI, 1583) $=$ Hymenochaete Kalchbrenneri Massee.

834. Peniophora laevigata (Fries) Massee (XXI, 1567) $=$ Xerocarpus Juniperi Karst.

835. Peniophora laevis (Fr.) . H. et L. $(\mathrm{XXI}, 1550)=$ Corticium calotrichum Karst.

836. Peniophora latitans (Karst.) v. H. et I. (XXI, 1554) = Corticium latitans Karst.

837. Peniophora Lycii (P.) v. H. et L. $(\mathrm{XXVIII}, 746)=$ Corticium rimosissimum Pass. 
838. Peniophora Molleriana (Bres.) Sace. (XXXII, 1092) $=$ Peniophora Roumeguèrii Bres.

839. Peniophora nuda (Fr.) Bres. (XXI, 155̄5; XXVIII, 788) $=$ Corticium mutabile Karst. in sched. = Corticium quercinum Fr. v. scutellatum Ellis.

840. Peniophora ochroleuca (Bres.) v. H. et L. (XXXII, 1107) $=$ Coniophora ochroleuca (Bres.)

841. Peniophora pubera (Fr.) Sacc. (XXVIII, 772, 789) $=$ Corticium flavidoalbum Cooke.

842. Peniophora radicata (P. H.) v. H. et L. (XXVIII, 746) $=$ Corticium radicatum $\mathrm{P}$. Henn.

843. Peniophora rimicola (Karst.) v. H. et L. (XXI, 15̄56) $=$ Corticium rimicolum Karst.

844. Peniophora serialis (Fr.) v. H. et L. (XXVIII, 777) $=$ Corticium Martianum B. et $\mathrm{C}$.

845. Peniophora setigera (Fr.) v. H. et L. (XXI, 1555, 1559; XXVIII, $742,791)=$ Corticium Chusqueae Pat. $=$ Corticium myxosporum Karst. = Lyomyces serus Karst. = Peniophora trachytricha E. et Ev.

846. Peniophora sordida (Schröt.) v. H. et L. (XXX, 4; XXXII, 1088) = Peniophora sordidella v. H. et L.

847. Peniophora sordida (Karst.) sensu Brinkm. (XXI, 15う5) $=$ ? Corticium sordidum Karst.

848. Peniophora sublaevis (Bres.) v. H. et L. (XXXII, 1088) $=$ Corticium sublaeve Bres.

849. Peniophora subsulphurea (Karst.) v. H. et L (XXI, 1561; XXXI, 9; XXXII, 1092) $=$ Corticium subsulphureum Karst. $=$ Corticium radicatum P. Henn.

8500. Peniophora subtilis (Schröt.) v. H. et L. (XXVIII, 837) $=H y$ pochnus subtilis Schröt.

851. Peniophora sulphurina (K.) v. H. et L. (XXI, 1573) = Tomentella sulphurina Karst.

852. Peniophora variegata (Roumg.) v. H. et L. (XXVIII, 766) $=$ Corticium variegatum Roumeg.

853. Peniophora velutina (D. O.) จ. H. et L. (XXVIII, 742; XXXII, $1082)=$ Corticium decolorans Karst. $=$ Corticium Eichlerianum Bres.

854. Peniophora viticola (Schw.) v. H. et L. (XXVIII, 779) $=$ Thelephora viticola Schw.

855. Perisporina manaosensis P. H. (XLIV, 906) ist eine Capnodiaceengattung mit Limacinia verwandt.

8566. Perisporiopsis Struthanthi P. H. (XLIV, 905) ist eine Capnodiaceengattung; Originalexemplar unreif.

857. Perisporium (Perisporiella) Myristicae P. Henn. (XLVIII, 408). Untergattung und Art zu streichen.

858. Perisporium typharum Sacc. (XIX, 1194) = Sporormia funiculorum Feltg.

859. Pestalozzia hypodermia (Niessl) v. H. (XXVI, 4) $=$ Sporidesmium hypodermium Niessl = Pestalozzia peregrina E. et $\mathrm{M}$. 
860. Pestalozziella longiseta (Rac.) v. H. (XXXVI, 891) = Neottiospora longiseta Rac.

861. Pezizella caespitulosa Bres. (XIX, 1265) $=$ Cenangium pallideflavescens Feltg. forma Atropae Feltg.

862. Pezizella lachnobrachya (Desm.) จ. H. $(\mathrm{XIX}, 1285)=P e z i z a$ lachnobrachya Desm.

863. Pezizella subaurantiaca Feltg. (XIX, 1277), Originalexemplar schlecht, za streichen.

864. Pezizella Teucrii Fckl. (XIX, 1268) = Mollisia luteo-fuscescens Feltg.

865. Phacidium coopertum (Desm.) v. H. $(\mathrm{XXVI}, 3)=$ Sphaeria cooperta Desm.

866. Phacidium Vincae Fckl. (XIX, 1261) = Pseudophacidium Vincae Feltg.

867. Phaeochora Chamaeropsis (Cooke) จ. H. (XXXVIII, 1513) = Auerswaldia Chamaeropsis Cooke.

868. Phaeoderris caespitosa (Niessl) v. H. (XXVI, 2) = Leptosphaeria caespitosa Niessl.

869. Phaeoderris (?) Heliopsidis (Schw.) ซ. H. (XXXVI, 876) $=$ Dothidea Heliopsidis Schw. $=$ Montugnella Heliopsidis (Schw.) Sace.

870. Phaeoderris ? salebrosa (Preuss) v. H. (XLVIII, 460) = Sphaeria salebrosa Preuss. (St. conid. est Plenodomus).

871. Phaeoderris (?) tumefaciens (E. et H.) v. H. (XXXVI, 876) $=$ Montagnella tumefaciens $\mathrm{E}$. et $\mathrm{H}$.

872. Phaeodomus Lauracearum v. H. (XXXVIII, 1529) ist der Conidienpilz von Auerswaldia Puttemansii P. H.

873. Phacohygrocybe P. Henn. (XLIV, 887) ist eine zweifelhafte Gattung.

874. Phaeoisaria gracilis (Vossel.) v. H. $(\mathrm{XXXV}, 414)=$ Isaria gracilis Vosselev.

875. Phaeoisaria sphecophila (Dittm.) จ. H. (XXXV, 414) = Isaria sphecophila Dittmar.

876. Phaeoisaria Sphingum (Schw.) v. H. (XXXV, 414) ist der Conidienpilz von Cordyceps Sphingum (Schw.).

877. Phaeoisaria surinamensis (Vossl.) v. H. $(\mathrm{XXXV}, 414)=$ Isaria surinamensis Vosseler.

878. Phaeopterula P. Henn. (XLVIII, 411) ist eine zweifelhafte Gattung.

879. Phaeoscutella Gynerii P. Henn. (XLVIII, 411) ist der Kot eines Insektes.

880. Phialea acuum (A. et S.) (XIX, 1290) = Phialea pertenera Feltg.

881. Phialea atro-sanguinea (Fuck.) v. H. (I, 21; XII, 187; XIII, 331) = Tapesia atro-sanguinea Fuck. = Patellea pseudosanguinea Rehm.

882. Phialea cyathoidea (Bull.) (XIX, 1291) = Phialea cyathoidea (Bull.) var. puberula Feltg.

883. Phialea dolosella Karst. (XIX, 1291) = Belonoscypha Dulcamarae Felty.

884. Phialea subpallida Rehm (XII, 187) $=$ ? Helotium subconfluens Bres.

885. Phialea Urticae (P.) $(\mathrm{XIX}, 1291)=$ Phialea vitigena Feltg. 
886. Phillipsiella Cooke 1878 (XXXV, 359) = Microphyma Speg. 1889.

887. Phillipsiella Ilicis (Ell.) จ. H. (XXXVII, 1229) = Asterina Ilicis Ell. = Henningsiella Ilicis (Ell.) v. H.

888. Phillipsiella Puiggari (Speg.) v. H. $(\mathrm{XXXV}, 350)=$ Microphyma Puiggari Speg.

889. Phleospora Robiniae (Lib.) จ. H. (XIII, 336) = Septoria Robiniae Desm. = Ascochyta Robiniae Lasch = Septosporium curvatum Rbh. = Septoria curvata Sacc. f. diversispora Faut. $=$ Fusarium Vogelii P. Henn.

890. Phomatospora hydrophila P. H. et K. (XIX, 1249) = Phomatospora secalina Feltg.

891. Phomopsis ramealis (Desm.) จ. H. (XLII, 647) = Sporonema rameale Desm. $=$ Phoma sambucina Sace. $=$ ? Phoma foetida Brun.

892. Phomopsis strobilina (Desm.) จ. H. (XLII, 647) = Sporonema strobilinum Desm.

893. Phragmographum Bactridis P. Henn. (XLVIII, 410) ist eine Flechte: Opegraphella Müll. Arg. spec.

894. Phragmonaevia (Naeviella) coeruleo-viridis (Rehm) v. H. (XLVIII, $381)=$ Ploettnera coeruleo-viridis (Rehm) P. Henn.

895. Phragmopeltis Siparunae P. H. (XLVIII, 393) ist der Conidienpilz von Polystomella? (Dothideaceae).

896. Phyllachora Cytharexyli (Rehm) v. H. (XXIX, 29) = Physalospora Cytharexyli Rehm.

897. Phyllachora Lauracearum (P. Henn.) จ. H. (XLIV, 930) = Pseudomelasmia Lauracearum P. Henn

898. Phyllachora Salaciae (P. Henn.) v. H. $(X L I V, 931)=$ Sirentyloma Salaciae P. H.

899. Phyllachora Scirpi Feltg. (XIX, 1258) ist zu streichen.

900. Phyllachora sp. (immatur.) (XLI, 430) = Rhytisma ustulatum Cooke.

901. Phyllachora Tjankorreh Rac. (XLVIII, 410) $=$ Lophiella Bambusae P. Henn.

902. Physalospora Alismatis Feltg. (XIX, 1200) ist zu streichen.

903. Physalospora Euryae (Rac.) จ. H. (XXXVI, 832) = Myocopron Euryae Racib,

904. Physalospora foliorum (Sacc.) v. H. (XXIII, 138) = Physalospora gregaria Sace. var. foliorum Sace. $=$ Physalospora gregaria Sace. var. Taxi Feltg.

905. Physalospora microspora Feltg. (XIX, 1200) ist zu streichen.

906. Physospora albida v. H. (XVII, 690) = Noematogonium album Bainier.

907. Picoa Carthusiana Tul. (XII, 187) = Picoa ophthalmospora Quel.

908. Piggotia Fraxini B. et Curt. (XLII, 631) = Dothichiza Libert (non Saccardo).

909. Piggotia Negundinis Ell. et D. (XLII, 631) = Dothichiza Libert (non Saceardo). 
910. Pilacre (Ecchyna) flavovirens (D. et M.) จ. H. (XLI, 393) = Lasioderma flavovirens $\mathrm{D}$. et $\mathrm{M}$.

911. Piptostomum domingense Lév. (XLII, 655) ist ein steriles Stroma.

912. Pirostoma Fries (XLII, 633) ist nach dem Typus eine zu streichende Gattung.

913. Pirostoma circinans Fries (XLII, 633) ist ein steriler Pilz, zu streichen.

914. Pirottaea longipila Feltg. (XXIX, 1275), Originalexemplar schlecht, zu streichen.

915. Pithomyces flavus Berk. et Br. (XLII, 668) = Neomichelia melaxantha Penz et Sacc.

916. Platygloea Eriophori (Bres.) จ. H. (XXXVII, 1157) = Kriegeria Eriophori Bres. $=$ Septogloeum dimorphum Sace.

917. Plectophoma bacteriospermum (Pass.) v. H. $(\mathrm{XXV}, 639)=P h y l$ losticta basteriosperma Pass.

918. Plenodomus Preuss (XLVIII, 460) ist eine stromatische Form, wenig verschieden von Phomopsis Sace.

919. Plenodomus Lingam (Tode) v. H. (XLVIII, 460) = Phoma Lingam (Tode) Desm. = Plenodomus Rabenhorstii Preuss.

920. Pleococcum Robergei Desm. (XLII, 655) ist ein steriler, unbestimmbarer Pilz.

921. Pleonectria coffeicola Zimm. (XLIV, 913) = Limacinula ? coffeicola (Z.) v. H.

922. Pleospora coronata Nssl. (XIX, 1223) = Pleospora leptosphaerioides Sacc. et Th. forma Oenotherae Feltg.

923. Pleospora Feltgeni S. et Syd. var. Pseud-Acori Feltg. (XIX, 1218) ist zu streichen.

924. Pleospora filicina Feltg. (XIX, 1218) ist zu streichen.

925. Pleospora herbarum (P.) (XIX, 1218, 1219, 1221, 1223, 1224; $\mathrm{XXV}, 615)=$ Cleistotheca papyrophila Zukal $=$ Pleospora collapsa Feltg. = Pleospora Convallariae Cocc. et Mor. $=$ Pleospora Convallariae Coce. et Mor. var. Polygonati Feltg. = Pleospora discoidea Feltg. = Pleospora herbarum (P.) var. sepincola Feltg. = Pleospora massarioides Feltg. $=$ Pleospora Negundis Oud. $=$ Pleospora Tiliae Feltg. = Pleospora Vitis Catt. forma Ribisalpini Feltg.

926. Pleospora herbarum Rabh. sensu Niessl (XXIII, 138) $=$ Pleospora discors Feltg. non $\mathrm{O}$. et de N. (Pleospora Feltgenii Sacc. et Syd.)

927. Pleospora infectoria Fekl. (XIX, 1219, 1222) = Pleospora Ribesia Feltg. = Pleospora socialis Nssl. forma Lilii Feltg. = Pleospora Clematidis Fckl. forma Viburni Feltg.

928. Pleospora juglandina Feltg. (XIX, 1223) = Pleospora infectoria Fckl. mit Pleospora herbarum (P.).

929. Pleospora Oenotherae Feltg. (XIX, 1224) ist zu streichen.

930. Pleospora opaca Wegelin (XIX, 1206, 1219) $=$ Pleospora Feltgenii Sace. et Syd. var. Eriophori Feltg. = Pleospora scabra Mouton $=$ Phorcys Eriophori Feltg. 
931. Pleospora rubicunda Nssl. (XIX, 1221, 1222) $=$ Pleospora culmigena Feltg. = Pleospora Glyceriae Feltg. = Pleospora lacustris Feltg.

932. Pleospora Sorghi Feltg. (XIX, 1222) ist zu streichen.

933. Pleospora vulgaris Nssl. (XIX, 1216) $=$ Strickeria Cerasi Feltg.

934. Pleurotus nidulans (P.) (XII, 188) = Claudopus odorativus Britz.

935. Ploettnera coeruleo-viridis (Rehm) $(\mathrm{XIX}, 1259)=$ Stictophacidium Rehmianum Feltg.

936. Plowrightia basirufa (B. et C.) v. H. (XXXVII, 1222) $=$ Dothidea basirufa Berk. et Curt.

937. Plowrightia Gastrolobii (P. H.) v. H. (XLVIII, 418) = Lizoniella Gastrolobii (P. Henn.) Sace.

938. Plowrighta Koordersii (P. H.) v. H. (XXXVII, 1214) $=$ Microcyclus Koordersii P. Henn.

939. Plowrightia Oxylobii (P. H.) จ. H. (XLVIII, 420) = Lizonia (Lizoniella) Oxylobii P. Henn.

940. Plowrightia Rhynchosporae (Rehm) จ. H. (XLVIII, 421)=Lizonia Rhynchosporae Rehm.

941. Pocosphaeria eriophora (Cke.) Berl. $(\mathrm{XIX}, 1211)=$ Acantho . stigma Heraclei Feltg.

942. Podaxon carcinomalis (L.) Fr. (XXIX, 16) = Podaxon mossamedensis Welw. et Curr. var. Emini P. Henn.

943. Podocrea adpropinquans (Cesati) v. H. (XXXV, 301) = ? Podocrea Solmsii Fischer.

944. Polycyclus Alsophilae (Rac.) v. H. (XXXVIII, 1542) $=$ Hysterostomella Alsophilae Rac.

945. Polycyclus andinus (Pat.) v. H. (XXXVII, 1542) $=$ Hysterostomella andina Pat.

946. Polycyclus filicina (Berk. et C.) จ. H. (XXXVIII, 1542) $=$ Rhytisma filicinum $\mathrm{B}$. et $\mathrm{Br}$. = Hysterostomella filicina (B. et $\mathrm{Br}$.) v. $\mathrm{H}$.

947. Polycyclus rhytismoides (Speg.) จ. H. (XXXVIII, 1542) = Hysterostomella rhytismoides Speg.

948. Polyporus albidus Trog. (XLVIII, 447) $=$ Polyporus Ptychogaster Ludwig.

949. Polyporus laccatus Kalchbr. (XVII, 688) = Ganoderma Pfeifferi Bres.

950. Polyporus Schweinitzii Fr. st. jun. $(\mathrm{X} \nabla I I, 688)=$ ? Merulius giganteus Sauter.

951. Polystomella Speg. $1888($ XXXVI, 848) $=$ Microcyclus Sace. et Syd. 1904.

952. Polystomella Abietis v. H. (XLI, 451) = Microthyrium pinastri Rehm, Ascom. exs. 1079.

953. Polystomella guaranitica (Speg.) จ. H. (XLI, 433) $=$ Schneepia guaranitica Speg.

954. Poria sanguinolenta (A. et S.) meo sensu nec Bres. (XXIII, 93; XXXV, 442) = Podoporia confluens Karst. (Syn. ined.).

955. Protodontia v. H. (XXIII, 83) = ? Protohydnum Möll. 
956. Psathyra subcernua (Schulz) v. H. (XXIII, 99) = Agaricus (Nolanea) subcernua Schulz $=$ Clitopilus conissans Peck (teste Bresad.).

957. Pseudomassaria Jacz. (VII, 41) = Aplacodina Rubl.

958. Pseudomeliola socia (P. H.) v. H. (XLI, 435) = Saccardomyces socius $\mathrm{P} . \mathrm{H} .=$ Ophionectria socia (P. H.) v. H.

959. Pseudonectria Bambusae (B. et Br.) v. H. (LII, 35̃4) = Nectria Bambusae Berk. et Broome 1873.

960. Pseudonectria callorioides (Rehm) v. H. (LII, 377) = Nectriella callorioides Rehm $1898=$ Pseudonectria tornata v. H. 1909 (forma).

961. Pseudonectria Strasseri (Rehm) Weese $(\mathrm{XL})=$ Nectria Strasseri Rehm 1907.

962. Pseudopatellina conigena (Niessl) v. H. (XXXIII, 1025) $=$ Dacrymyces conigenus Niessl $=$ ? Achitonium strobilicala Kalchbr.

963. Pseudophacidium Rehmii (Feltg.) v. H. (XIX, 1264) = Cenangium Rehmii Feltg.

964. Pseudorhynchia polyrhyncha (P. et S.) v. H. (XXXVII, 1206) $=$ Ceratostomella polyrhyncha $\mathrm{P}$. et $\mathrm{S}$.

965. Pseudosphaerella Baccharidis (Rehm) v. H. (XLVIII, 425) $=$ Lizonia Baccharidis Rehm.

966. Pseudosphaerella Cupaniae (Rehm) v. H. (XLVIII, 426) $=$ Lizonia (Lizoniella) Cupaniae Rehm.

967. Pseudosphaeria callista (Rehm) v. H. (XXIII, 129; XXV, 635) = Sphaerulina callista Rehm.

968. Pseudosphaeria pachyasca (Niessl) v. H. (XXV, 635) $=$ Leptosphaeria pachyasca Niessl.

969. Pseudostictis Lambotte (VII, 49) ist eine zu streichende Gattung.

970. Psilopezia tremellosa Hazsl. (XXXV, 399) = Psilopezia Fleischeriana P. H. et E. N. = Pillopezia Pauli P. Henn. = ? Peziza hydrophila Peck. = ? Psilopezia Mölleriana P. Henn.

971. Pucciniostele Elettariae (Rac.) v. H. (XLI, 228) $=$ Schröteriaster Elettariae Rac. Nach Dietel in litt. nur Uredo.

972. Puttemansia P. Henn. 1902 (XLVIII, 405) = Scoleconectria Seaver 1909.

973. Puttemansia albolanata (Speg.) v. H. (XXXVI, 824) = Paranectria (?) albolanata Speg.

974. Puttemansia lanosa P. Henn. 1902 (XLIV, 899) = Calonectria mit Stroma $=$ Scoleconectria Seaver 1909.

975. Puttemansiella Desmodii P. Henn. (XLVIII, 439) = Dermatea? steril.

976. Pyrenopeziza Alismatis Feltg. (XIX, 1273), Originalexemplar schlecht, zu streichen.

977. Pyrenopeziza Noppeyana (Feltg.) v. H. $(\mathrm{XIX}, 1287)=$ Lachnum Noppeyanum Feltg.

978. Pyrenopeziza Polygonati (Feltg.) v. H. (XIX, 1276) $=$ Beloniella Polygonati Feltg.

979. Pyrenopesiza Rhinanthi (Sommf.) Sace. (I, 33; XVII, 654) $=$ Sphaeria complanata Tde. pro part. = Sphaeria Rhinanthi Sommerf. = Sphaeronema Rhinanthi Lib. $=$ Zythia Rhinanthi 
Fr. = Phoma deustum Fekl. = Mollisia Rhinanthi Karst. = Doassansia Rhinanthi Lagh.

980. Pyrenophora hispida N. (XIX, 1225) = Pyrenophora flavo-fusca Feltg.

981. Pyrenophora Salsolae Gr. var. Majanthemi Feltg. (XIX, 1224) ist zu streichen.

982. Pyrenotrichum aeruginosum v. H. (XXXVIII, 1530) = Tricho. sperma aeruginosa v. H.

983. Pyrenotrichum cyphelloideum จ. H. (XXIII, 145) $=$ Trichosperma cyphelloidea v. H.

984. Pyrenotrichum ? Woodsianum (Sace. et Berl.) v. H. (XLII, 637) $=$ Melophia Woodsiana Sace. et Berl.

985. Radulum (pallidum B. et O. ?) (XXVIII, 783) = Corticium colliculosum B. et $\mathrm{C}$.

986. Radulum quercinum Fries (XXI, 1566) $=$ ? Xerocarpus Corni Karst.

987. Ramularia Alismatis Fautr. (XI, 25) = Didymaria aquatica Starb.

988. Ramularia Anagallidis Lindr. (XII, 189) = Ramularia nivea $\mathrm{K}$. et $\mathrm{B}$.

989. Ramularia Anchusae Mass. (I, 57) = Ramularia Anchusac-offcinalis Elias.

990. Ramularia aromatica (Sace.) v. H. (XI, 26) = Septocylindrium aromaticum Sace.

991. Ramularia Carthusiana (Sace.) v. H. (XXXVII, 1236) = Septogloeum Carthusianum Sace.

992. Ramularia Cupulariae Pass. (XII, 189) = Ovularia Inulae Sace. = Ramularia Inulae brittanicae Allesch.

993. Ramularia Gei (Elias.) v. H. (VII, 57) = Ovularia Gei Elias $=$ Ramularia submodesta $\mathrm{v}$. H.

994. Ramularia Levistici Oud. (III, 177) = Cylindrospora Levistici Schroet. $=$ Ramularia Vestergreeniana Allesch.

995. Ramularia nivea (Ung.) (XII, 189) = ? Ramularia Beccabungae Faut.

996. Rebentischia unicaudata (B. et Br.) (XIX, 1226; XXIII, 137) $=$ Rebentischia thujana Feltg. = Lophiostoma caulium $\mathrm{C}$. et de N. f. Vitalbae Feltg.

997. Rehmiomyces Pouroumae P. Henn. (XLVIII, 379) ist eine typische Patellariacee.

998. Rhamphoria delicatula Nssl. (XIX, 1207) $=$ Zignoëlla prorumpens (Rehm) var. oxystoma Feltg.

999. Rhamphoria occultata (Feltg.) v. H. (XIX, 1197) = Ceratosphaeria occultata Feltg.

1000. Rhamphoria pyriformis (Fries) v. H. (LIII) $=$ Sphaeronaema pyriforme Fr. $1823=$ Rhamphoria thelocarpoidea v. H. 1906.

1001. Rhamphoria thelocarpoidea v. H. (XXIII, 124) = Coronophora thelocarpoidea v. H.

1002. Rhamphoria tympanidispora Rehm (XIX, 1196) $=$ Ceratosphaeria obliquata Feltg. 
1003. Rhynchonectria จ. H. (I, 37; XXXIII, 1026) = Eleutherosphaera Grove (1907).

1004. Rhynchonectria longispora (Ph. et Pl.) v. H. (I, 37) $=$ Eleutheromyces longisporus $\mathrm{Ph}$. et $\mathrm{Pl}$.

1005. Rhytisma Astrocaryi Mont. (XLI, 433) ist zu streichen.

1006. Rosellinia conglobata F(kl. $(\mathrm{XIX}, 1197)=$ Rosellinia occultata Feltg.

1007. Rosellinia conglobata (Fck.) var. microtricha (Feltg.) v. H. $(\mathrm{XIX}, 1198)=$ Rosellinia sordaria (Rehm) var. microtricha Feltg.

1008. Rosellinia culmorum (Feltg.) จ. H. ad. inter. (XIX, 1212) $=$ Trichosphaeria culmorum Feltg.

1009. Rosellinia Goliath (Speg.) จ. H. (XXIX, 23) = Hypoxylon Goliath Speg.

1010. Rosellinia ligniaria (Grev.) $(\mathrm{XIX}, 1198)=$ Rosellinia brassicaecola Feltg.

1011. Rosellinia (Tassiella) Miconiae (P. Henn.) v. H. (XXXVI, 828) $=$ Auerswaldia Miconiae P. H.

1012. Rostafinstia austratis Speg. (XXXVI, 899) ist ein steriles Mycel (nach Spog. zu Rosellinia australis Speg.).

1013. Roussoëlla Bauhiniae (Wint.) v. H. (LII, 392) = Trabutia Bau. hiniae Wint.

1014. Rutströmia firma Karst. (XIX, 1286) $=$ Rutströmia firma Karst. var. acuum Feltg.

1015. Saccardinula myrticola Rehm (XLVIII, 416) ist eine Flechte: Phyllobathelium Müll. ?

1016. Saccardinula (?) Rickii (Rehm) จ. H. (XXXV, 372) = Saccardia Durantae P. et Lag. var. Rickii Rehm.

1017. Sacidium ?? brasiliense Speg. (XLII, 657) = Pilobolus-Sporangium nach dem Originalexemplar.

1018. Sacidium chartarum Sace. et Penz. (XLII, 656) $=$ PilobolusSporangium.

(Fortsetzung folgt.)

\section{Literatur - Übersicht ${ }^{1}$ ).}

Juli und Angust 1913.

Baudy š E. Pro Čechy nove hólky. (Neue Cecidien für Böhmen.) Sbornik klubu prírodovédeckého $\mathrm{v}$ Praze für das Jahr 1912. $16 \mathrm{~S}$. Prag, 1913. $8^{0}$. Mit 4 Textabb.

Busich E. Die endotrophe Mykorhiza der Asclepiadaceae. (Verbandl. d. zoolog.-botan. Gesellsch. Wien, LXIII. Bd., 1913, 5. v. 6. Heft, S. 240 bis 264.) $8^{\circ}$.

1) Die „Literatur-Übersicht“ strebt Vollständigkeit nur mit Rüeksicht auf jene Abhandlungen an, die entweder in Österreich erscheinen oder sich anf die Flora dieses Gebietes direkt oder indirekt beziehen, ferner auf selbständige Werke des Auslandes. Zur Erzielung tunlichster Vollständigkeit werden die Herren Autoren und Verleger um Einsendung von neu erschienenen Arbeiten oder wenigstens am eine Anzeige über solche böflichst ersucht. Die Redaktion. 